\title{
COVID-19 Detection in Chest X-ray Images using a Deep Learning Approach
}

\author{
Fátima A. Saiz*, Iñigo Barandiaran
}

Vicomtech Foundation, Basque Research and Technology Alliance (BRTA), Donostia - San Sebastián (Spain)

Received 10 April 2020 | Accepted 29 April 2020 | Published 30 April 2020

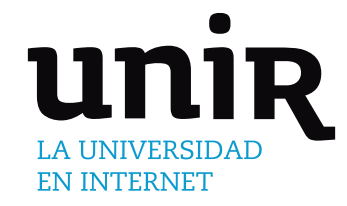

\section{ABSTRACT}

The Corona Virus Disease (COVID-19) is an infectious disease caused by a new virus that has not been detected in humans before. The virus causes a respiratory illness like the flu with various symptoms such as cough or fever that, in severe cases, may cause pneumonia. The COVID-19 spreads so quickly between people, affecting to 1,200,000 people worldwide at the time of writing this paper (April 2020). Due to the number of contagious and deaths are continually growing day by day, the aim of this study is to develop a quick method to detect COVID-19 in chest X-ray images using deep learning techniques. For this purpose, an object detection architecture is proposed, trained and tested with a public available dataset composed with 1500 images of non-infected patients and infected with COVID-19 and pneumonia. The main goal of our method is to classify the patient status either negative or positive COVID-19 case. In our experiments using SDD300 model we achieve a $94.92 \%$ of sensibility and $92.00 \%$ of specificity in COVID-19 detection, demonstrating the usefulness application of deep learning models to classify COVID-19 in X-ray images.

\section{KEYWORDS}

COVID-19, Deep

Learning, Object

Detection, X-ray.

DOI: $10.9781 /$ ijimai.2020.04.003

\section{INTRODUCTION}

$\mathrm{T}$ HE new SARS-CoV-2 coronavirus, which produces the disease known as COVID-19, kept the whole world on edge during the first months of 2020. It provoked the borders close of many countries and the confinement of millions of citizens to their homes due to infected people, which amounts to 868,000 confirmed cases worldwide at this moment (April 2020). This virus was originated in China in December 2019. From March 2020, Europe was the main focus of the virus sprout, achieving more than 445,000 infected people.

China, with a total of 3,312 deaths and more than 81,000 infected people, has managed to contain the virus almost three months after the start of the crisis in December 2019. Italy, which surpassed the Asian country in death toll on March 2020, became the most affected country, in number of deceased is followed by Spain, with more than 10,000 dead based on a report made on April 2020. This number was constantly growing. There were different studies that predicted the growth of the curves of infections, based on different parameters such as exposed, infected or recovered human's number. These studies allowed to get an idea of the transmission dynamics that could occur in each country [1] [2].

The origin of the outbreak is unknown. The first cases were detected in December 2019. The clinical characteristics of COVID-19 include respiratory symptoms, fever, cough, dyspnea, and viral pneumonia [3] [4]. The main problem of these symptoms is that there are virusinfected asymptomatic patients.

\footnotetext{
* Corresponding author.

E-mail address: fsaiz@vicomtech.org
}

The test to detect the COVID-19 is based on taking samples from the respiratory tract. It is carried out by a health care professional at home, generally when the case study is asymptomatic or symptoms are mild, or in a health center or hospital, if the patient is admitted for a serious condition. Carrying out as many tests as possible has shown to be the key tool to stop the virus in countries like Germany or South Korea. Spain was not able to carry out so many tests, therefore it is important to research and develop alternative methods to perform these tests in a quick and effective way.

AI and radiomics applied to X-Ray and Computed Tomography (CT) are useful tools in the detection and follow-up of the disease [5] [6]. As stated in [7], conspicuous ground grass opacity lesions in the peripheral and posterior lungs on CT images are indicative of COVID-19 pneumonia. Therefore, CT can play an important role in the diagnosis of COVID-19 as an advanced imaging evidence once findings in chest radiographs are indicative of coronavirus. AI algorithms and radiomics features derived from Chest X-rays would be of huge help to undertake massive screening programs that could take place in any country with access to X-ray equipment and aid in the diagnosis of COVID-19 [8] [9].

The current situation evokes the necessity to implement an automatic detection system as an alternative diagnosis option to prevent COVID-19 spreading among people. There are different studies that apply machine learning for this task, such as Size Aware Random Forest method (iSARF) that was proposed by [10], in which subjects were categorized into groups with different ranges of infected lesion sizes. Then a random forest-based classifier was trained with each group. Experimental results show that their proposed method yielded an accuracy of 0.879 under five-fold cross-validation, a sensitivity of 0.907 and a specificity of 0.833 . 
Deep learning techniques are also used in order to achieve better results than using more traditional machine learning approaches. One of the most used approach in image classification is the use of convolutional neural networks (CNNs). This type of models are used in different studies for COVID-19 detection in medical images like in [11], in their study the authors propose a CNN model trained with a randomly selection of image regions of interest (ROIs), achieving a $85.2 \%$ of accuracy, 0.83 of specificity and 0.67 of sensitivity. Other example of the results that can be obtained using CNNs is presented by [12]. They propose the COVID-Net CNN network obtaining $92.4 \%$ of accuracy, $80 \%$ of sensibility and $88.9 \%$ of specificity.

A method called COVIDX-Net is presented by [13], COVIDXNet includes seven different architectures of deep convolutional neural network models, such as a modified version of Visual Geometry Group Network (VGG19) and the second version of Google MobileNet. Each deep neural network model is able to analyze the normalized intensities of the X-ray image to classify the patient status either negative or positive COVID-19 case. Their experiments evaluation achieves f1-scores of 0.89 and 0.91 for healthy and COVID-19 detection respectively.

The results shown in mentioned works demonstrate that deep learning techniques are useful for the virus detection, and that improve the obtained metrics using more traditional machine learning approaches [11] [12] [13].

The main contribution of our paper is focused on the improvement of the detection accuracy of COVID-19, by proposing a new dataset that combines COVID-19 and pneumonia images to make more stable predictions and by applying image processing that allows imagestandardization and also improves model learning.

\section{METHOD}

We propose to use a deep convolutional neural network specialized for object detection along with a new dataset composed of COVID-19 and pneumonia images. Both are publicly available on GitHub [14] and Kaggle [15] respectively. The chest X-ray or CT images that are available in GitHub belong to COVID-19 cases. It was created by assembling medical images from public available websites and publications. This dataset contains 204 COVID-19 X-ray images. On the other hand, the Kaggle dataset was created for a pneumonia detection challenge. The images have bounding boxes around diseased areas of the lung. Samples without bounding boxes are negative and contain no definitive evidence of pneumonia. Samples with bounding boxes indicate evidence of pneumonia.

We propose a new dataset by merging COVID-19 and pneumonia images to obtain a wider and diverse one. The fact of having pneumonia images in the training dataset supposes an extra advantage, due to normal pneumonia and COVID-19 have similar appearance in chest $\mathrm{X}$-ray images. This dataset merge allows to get a robuster model that is able to better distinguish between those diseases. Another advantage of this merge is the fact of enlarging the train dataset, because the COVID-19 images are not abundant at the time of writing this paper. This merge does not enlarges COVID-19 image set but improves detection quality because of the similarity between pneumonia and COVID-19. Train with pneumonia images gives an extra knowledge to the model in order to not confuse COVID-19 with pneumonia, being more effective and stable in disease detection.

We split the images in train and test sets, dividing all the data in a balanced way, meaning that all samples of each class in the training sets are well-balanced, in order to avoid biased results. For this purpose, even though we have a large number of pneumonia and normal images, we compose a dataset of 1500 images.
We compose the dataset as follows: we select 104 COVID-19 images, 205 health lung images and 204 pneumonia images for training, and 100 COVID-19 images, 444 health lung images and 443 pneumonia images for testing. In the Kaggle dataset there are more samples of pneumonia and normal images, but we select only 205 for training with the purpose of having a balanced dataset. In the test stage, we add more pneumonia and normal images to demonstrate the robustness of the model giving no false positives in the detection of COVID-19. Summing up, we use a train set composed of 513 images and a test set of 887 images in total.

In the next step, training images are labeled using a XML annotation file based on Pascal VOC format [16]. Each sample in the dataset is an image with ground truth bounding boxes for each object in the images as shown in the Fig. 1.

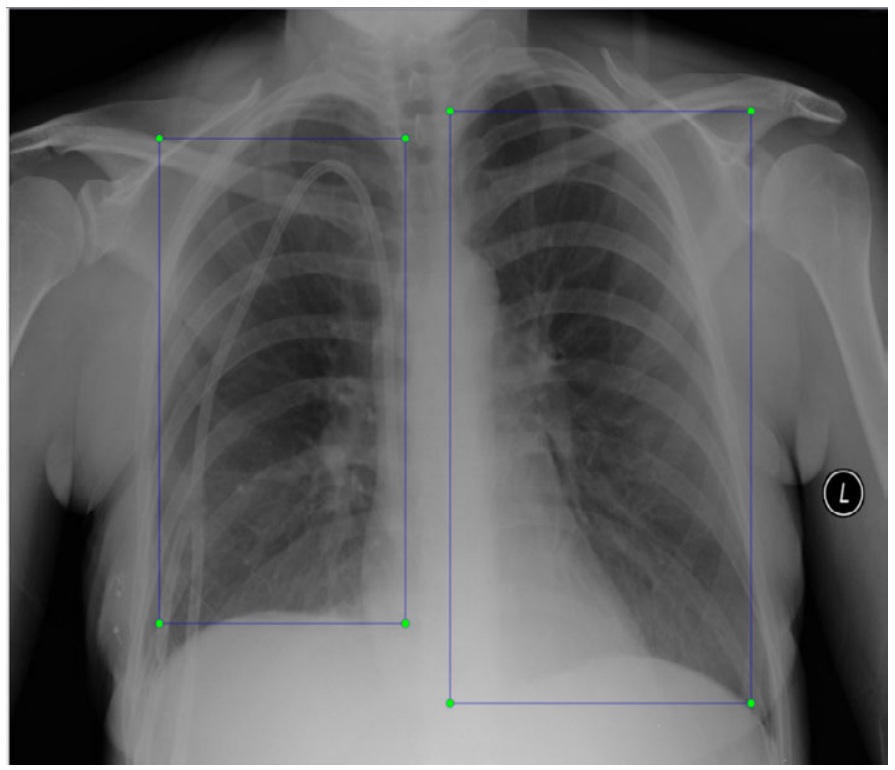

Fig. 1. Created bounding boxes of normal regions in a chest X-Ray image.

\section{MODEL ARCHITECTURE}

The selection of the used architecture is based on the good results obtained with CNNs in the state-of-the-art works for COVID-19 image classification, and the good results obtained in other similar tasks with this kind of architecture [11] [12] [13]. We used the same network architecture as proposed in [17], based on Single Shot Multibox Detector (SSD). This architecture is optimized for detecting objects in images using a single deep neural network. This approach discretizes the output space of bounding boxes into a set of default boxes over different aspect ratios and scales per feature map location. At prediction time, the network generates scores for the presence of each object category in each default box and produces adjustments to the box to better match the object shape. Additionally, the network combines predictions from multiple feature maps with different resolutions to naturally handle objects of various sizes.

Experimental results on different remarkable datasets confirm that SSD has comparable accuracy to methods that utilize more than one architecture for detecting objects being much faster, while providing a unified framework for both training and inference. Compared to other single stage methods, SSD has much better accuracy, even with a smaller input image size [17].

We use VGG-16 [18] as the base network for performing feature extraction in this architecture. This model is also based on Fast R-CNN. During training, we have multiple boxes with different sizes 
and different aspect ratios across the whole image. SSD finds the box that has more Intersection-Over-Union (IoU) compared with the ground truth. A detailed description of the layers architecture of SDD network is shown in [17].

Our main goal is to obtain a more robust model to various input object sizes and shapes. Therefore, during the SSD training a data augmentation step is performed. This process is composed by the following operations applied to every image in the dataset:

- Use the entire original input image.

- Sample a patch so that the minimum overlap with the objects is 0.1 , $0.3,0.5,0.7$, or 0.9 . The size of each sampled patch is a percentage between $[0.1,1]$ of the original image size.

- Randomly sample a patch.

During the inference, SDD uses 8732 boxes for a better coverage of object location. After the inference step, a set of boxes representing the detected objects are given along with the respective label and score, as shown in Fig. 2.

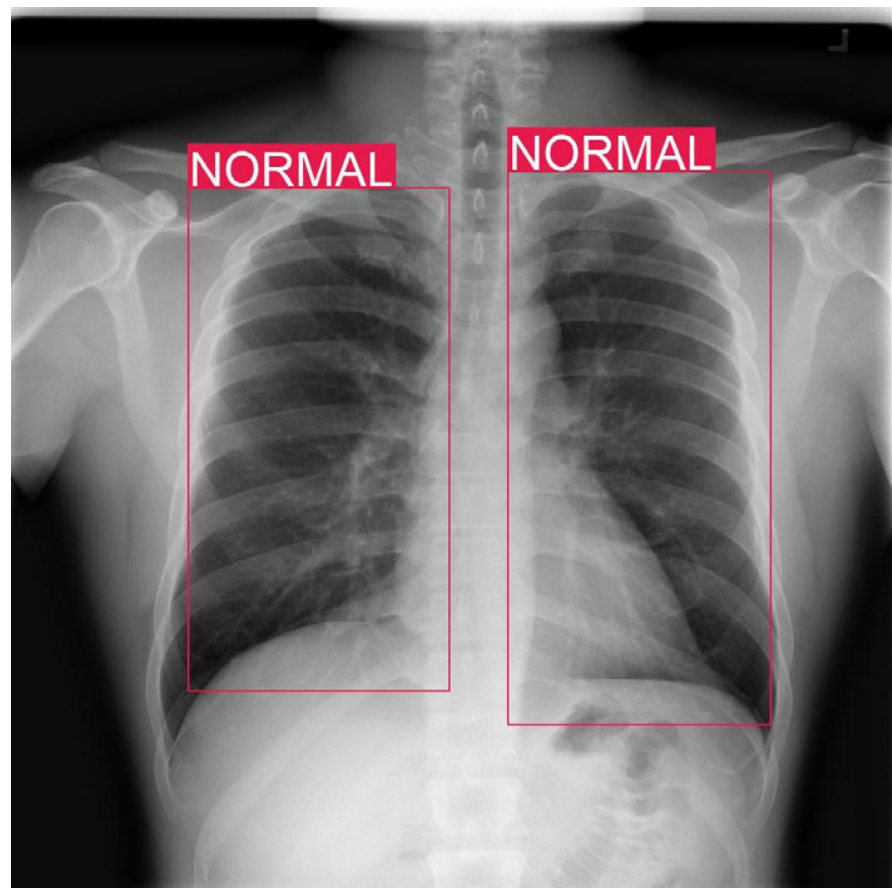

Fig. 2. Detection boxes of SSD model inference output detecting normal lungs.

One of the advantages of this model is the possibility of precise object localization, which is not the case in previous works [11] [12] [13].

\section{EXPERIMENTS AND RESULTS}

The first experiment made in this work, is the contrast adjustment of each image in the dataset. This adjustment is necessary because the exposure time in X-Ray images can be different between acquisitions. All the images of the dataset are from different hospitals around the world, so the image acquisition settings and conditions are different in each place. In X-Ray images, an adjustment in the voltage spike results in a change in the contrast of the radiography. Exposure time, which refers to the time interval during which $\mathrm{x}$-rays are produced, is also a factor that affects the contrast of the obtained image [19].

In order to get image similarity between the dataset, Contrast Limited Adaptive Histogram Equalization (CLAHE) [20] is applied. This is a transformation that aims to obtain a histogram with an even distribution for an image. That is, there is the same number of pixels for

each level of gray in the histogram of a monochrome image. As cited in [20], in X-ray imaging, when continuous exposure is used to obtain an image sequence or video, usually low-level exposure is administered until the region of interest is identified, so reducing the radiation applied to the patients. As a drawback, images with low signal-to-noise ratio are obtained. In this case, and many other similar situations, it is desirable to improve image quality by using some type of image enhancement such as histogram equalization algorithms. An example of the application of this image operation is shown in Fig. 3.

ORIGINAL
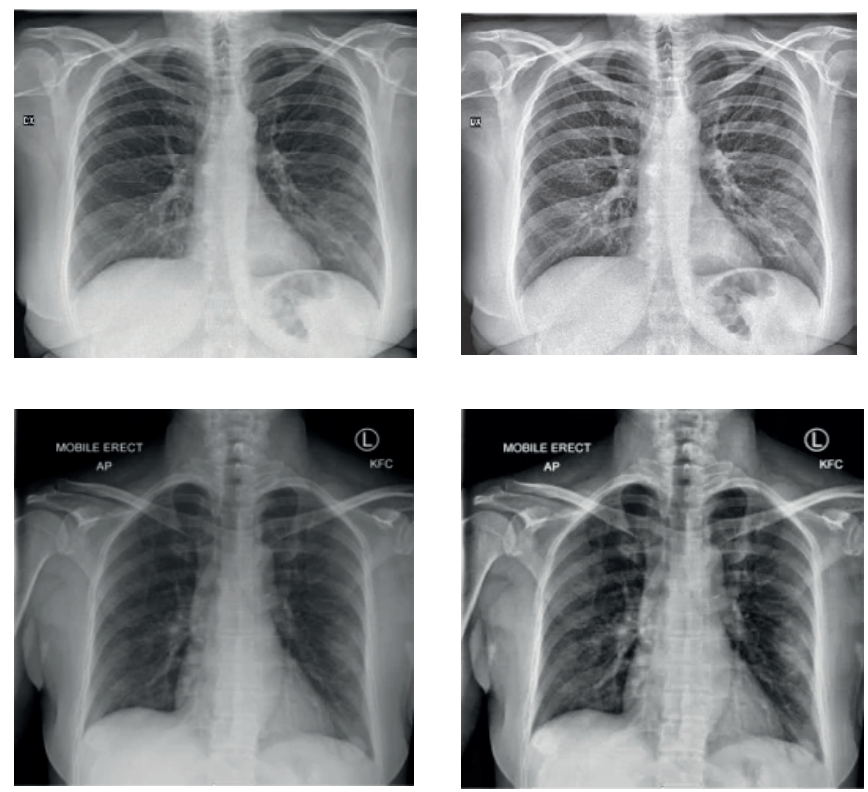

Fig. 3. Comparison between original image and CLAHE applied images.

The differences obtained in the detection applying or not CLAHE in the train and test datasets are shown in TABLE I. As the table shows, the fact of applying this pre-processing increases notably the detection accuracy in health and infected lungs.

We load VGG-16 weights trained on ImageNet. There are many works that evaluate the accuracy improvement using transfer learning, specially in small datasets [21] [22]. We apply these weights because though lower layers learn features that are not necessarily specific to this dataset, this action improves the detection accuracy and the sensibility and specificity metrics. The key idea is to take advantage of a trained model for similar images and adapt a base-learner to a new task for which only a few labeled samples are available.

The last experiment evaluates the detection accuracy obtained in the detection of COVID-19. For this purpose, we set apart two sets of images, one with non-COVID-19 and the other one with COVID-19 positives. We obtain different metrics that can be seen in TABLE II. Another metric that we take in care is the inference time that we obtain running the model on a GPU, achieving 0,13 s per image.

TABLE I. Obtained Results in Image Classification Applying or Not CLAHE IN THE DATASET.

\begin{tabular}{ccccc}
\hline Image class & CLAHE & Total images & True detection & Accuracy \\
\hline Normal & No & 887 & 827 & $93.24 \%$ \\
Normal & Yes & 887 & 842 & $\mathbf{9 4 . 9 2 \%}$ \\
COVID-19 & No & 100 & 83 & $83.00 \%$ \\
COVID-19 & Yes & 100 & 92 & $\mathbf{9 2 . 0 0 \%}$ \\
\hline
\end{tabular}


TABLE II. Obtained Metrics Values

\begin{tabular}{ccc}
\hline Metric & Operation & Value \\
\hline Sensibility & $842 /(842+45)$ & 0.9492 \\
Specificity & $92 /(92+8)$ & 0.9200 \\
\hline
\end{tabular}

\section{Conclusion}

This study demonstrates the useful application to detect COVID-19 in chest X-ray images based on image pre-processing and the proposed object detection model.

The proposed merged dataset using pneumonia images allows getting a more robust model that is able to distinguish between COVID-19 and pneumonia diseases. With the histogram equalization operation, we can get a normalized dataset that helps to model training step. It also improves the normal image detection and minimizes the false positives rate.

With our proposed method, we achieve a $94.92 \%$ of sensibility and $92.00 \%$ of specificity in COVID-19 detection. The detection accuracy obtained using this architecture and the proposed dataset improves the results described in [11] [12] [13]. These results demonstrate that object detection models trained with more images of similar diseases and applying transfer learning, combined with CLAHE algorithm for image normalization, could be successful in medical decision-making processes related with COVID-19 virus diagnosis.

\section{REFERENCES}

[1] M. Dur-e-Ahmad and M. Imran, "Transmission Dynamics Model of Coronavirus COVID-19 for the Outbreak in Most Affected Countries of the World," International Journal of Interactive Multimedia and Artificial Intelligence, vol. In Press, no. In Press, pp. 1-4, 2020.

[2] S. J. Fong, N. D. G. Li, R. Gonzalez-Crespo and E. Herrera-Viedma, "Finding an Accurate Early Forecasting Model from Small Dataset: A Case of 2019-nCoV Novel Coronavirus Outbreak," International Journal of Interactive Multimedia and Artificial Intelligence, vol. 6, no. 1, pp. 132140, 2020.

[3] Q. Li, X. Guan, P. Wu, X. Wang, L. Zhou, Y. Tong, R. Ren, K. S. M. Leung, E. H. Y. Lau, J. Y. Wong and others, "Early transmission dynamics in Wuhan, China, of novel coronavirus-infected pneumonia," New England Journal of Medicine, 2020.

[4] D. Wang, B. Hu, C. Hu, F. Zhu, X. Liu, J. Zhang, B. Wang, H. Xiang, Z. Cheng, Y. Xiong, Y. Zhao, Y. Li, X. Wang and Z. Peng, "Clinical Characteristics of 138 Hospitalized Patients With 2019 Novel Coronavirus-Infected Pneumonia in Wuhan, China," JAMA, vol. 323, pp. 1061-1069, 32020.

[5] S. Chauvie, A. De Maggi, I. Baralis, F. Dalmasso, P. Berchialla, R. Priotto, P. Violino, F. Mazza, G. Melloni and M. Grosso, "Artificial intelligence and radiomics enhance the positive predictive value of digital chest tomosynthesis for lung cancer detection within SOS clinical trial," European Radiology, p. 1-7, 2020.

[6] G. Chassagnon, M. Vakalopoulou, N. Paragios and M.-P. Revel, "Artificial intelligence applications for thoracic imaging," European Journal of Radiology, vol. 123, p. 108774, 2020.

[7] F. Song, N. Shi, F. Shan, Z. Zhang, J. Shen, H. Lu, Y. Ling, Y. Jiang and Y. Shi, "Emerging 2019 Novel Coronavirus (2019-nCoV) Pneumonia," Radiology, vol. 295, pp. 210-217, 2020.

[8] J. C. L. Rodrigues, S. S. Hare, A. Edey, A. Devaraj, J. Jacob, A. Johnstone, R. McStay, A. Nair and G. Robinson, "An update on COVID-19 for the radiologist-A British society of Thoracic Imaging statement," Clinical Radiology, 2020

[9] J. Wu, J. Liu, X. Zhao, C. Liu, W. Wang, D. Wang, W. Xu, C. Zhang, J. Yu, B. Jiang and others, "Clinical characteristics of imported cases of COVID-19 in Jiangsu province a multicenter descriptive study," Clinical Infectious Diseases, 2020.

[10] F. Shi, L. Xia, F. Shan, D. Wu, Y. Wei, H. Yuan, H. Jiang, Y. Gao, H. Sui and D. Shen, Large-Scale Screening of COVID-19 from Community
Acquired Pneumonia using Infection Size-Aware Classification, arXiv preprint arXiv:2003.09860, 2020.

[11] S. Wang, J. M. Bo Kang, X. Zeng and M. Xiao, "A deep learning algorithm using CT images to screen for Corona Virus Disease COVID-19)," medRxiv, 2020.

[12] L. Wang and A. Wong COVID-Net A Tailored Deep Convolutional Neural Network Design for Detection of COVID-19 Cases from Chest Radiography Images, arXiv preprint arXiv:2003.09871,2020.

[13] E. E.-D. Hemdan, M. A. Shouman and M. E. Karar, COVIDX-Net A Framework of Deep Learning Classifiers to Diagnose COVID-19 in X-Ray Images, arXiv preprint arXiv: arXiv:2003.11055, 2020.

[14] J. P. Cohen, P. Morrison and L. Dao, COVID-19 Image Data Collection, arXiv preprint arXiv: arXiv, arXiv:2003.11597, 2020.

[15] RSNA Pneumonia Detection Challenge. Kaggle. [online] Available at: $\quad$ https://www.kaggle.com/c/rsna-pneumonia-detection-challenge, Accessed 29 April 2020.

[16] M. Everingham, L. Van Gool, C. K. I. Williams, J. Winn and A. Zisserman, "The pascal visual object classes (voc) challenge," International journal of computer vision, vol. 88, p. 303-338, 2010.

[17] W. Liu, D. Anguelov, D. Erhan, C. Szegedy, S. Reed, C.-Y. Fu and A. C. Berg, "SSD Single Shot MultiBox Detector," Lecture Notes in Computer Science, p. 21-37, 2016.

[18] K. Simonyan and A. Zisserman, "Very Deep Convolutional Networks for Large-Scale Image Recognition," CoRR, vol. abs1409.1556, 2014.

[19] H. Jansen, Radiología dental. Principios y técnicas., Mc Graw Hill, 2002.

[20] A. M. Reza, "Realization of the contrast limited adaptive histogram equalization (CLAHE) for real-time image enhancement," Journal of VLSI signal processing systems for signal, image and video technology, vol. 38, p. 35-44, 2004.

[21] H.-W. Ng, V. D. Nguyen, V. Vonikakis and S. Winkler, "Deep learning for emotion recognition on small datasets using transfer learning," in Proceedings of the 2015 ACM on international conference on multimodal interaction, 2015.

[22] Q. Sun, Y. Liu, T.-S. Chua and B. Schiele, "Meta-transfer learning for few-shot learning," in Proceedings of the IEEE Conference on Computer Vision and Pattern Recognition, 2019.

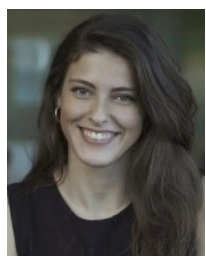

Fátima A. Saiz

Fátima Aurora Saiz Álvaro studied Engineering in Geomatics and Topography the University of the Basque Country during 2012-2017, being the first of its promotion. She obtained the title with her final degree project "Applications of programming languages to artificial intelligence, complex geophysical calculations and augmented reality" valued with honors, joining in the Talentia program of the Bizkaia Provincial Council. She completed the Master in Visual Analytics and Big Data of the Faculty of Engineering of the International University of La Rioja during 2017-2018. She obtained the title with her work "Study of architectures for the extraction and exploitation of superficial defects data using Deep Learning techniques". Since October 2017 she has been part of the Vicomtech staff as a researcher in the Department of Industry and Advanced Manufacturing, working in the field of cognitive vision and Deep Learning.

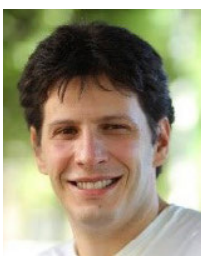

\section{Î́nigo Barandiaran}

Iñigo Barandiaran received his $\mathrm{PhD}$ in Computer Science from the University of the Basque Country in the fields of computer vision and pattern recognition. He worked as a researcher in the group of "Artificial intelligence and computer science" at the University of Basque Country. Since 2003 he is a researcher at Vicomtech, participating and leading various $R \& D$ national and international projects, in various sectors such as biomedicine and industry. He has several scientific publications in conferences and journals in areas such as image analysis, or pattern recognition. He is currently the director of the Industry and Advanced Manufacturing department at Vicomtech. 